\title{
Wronskian Determinant Solutions for the (3 + 1)-Dimensional Boiti-Leon-Manna-Pempinelli Equation
}

\author{
Hongcai Ma, Yongbin Bai \\ Department of Applied Mathematics, Donghua University, Shanghai, China \\ Email: hongcaima@hotmail.com
}

Received August 4, 2013; revised September 4, 2013; accepted October 1, 2013

Copyright (C) 2013 Hongcai Ma, Yongbin Bai. This is an open access article distributed under the Creative Commons Attribution License, which permits unrestricted use, distribution, and reproduction in any medium, provided the original work is properly cited.

\begin{abstract}
In this paper, we consider $(3+1)$-dimensional Boiti-Leon-Manna-Pempinelli equation. Based on the bilinear form, we derive exact solutions of $(3+1)$-dimensional Boiti-Leon-Manna-Pempinelli (BLMP) equation by using the Wronskian technique, which include rational solutions, soliton solutions, positons and negatons.
\end{abstract}

Keywords: (3 + 1)-Dimensional Boiti-Leon-Manna-Pempinelli Equation; The Wronskian Technique; Soliton; Negaton; Positon

\section{Introduction}

The Wronskian technique is introduced by Freeman and Nimmo [1]. After that, many researches are based on the Wronskian technique.

The $(2+1)$-dimensional BLMP equation was first derived in [2]:

$$
u_{y t}+u_{x x x y}-3 u_{x x} u_{y}-3 u_{x} u_{y}=0
$$

where $u=u(x, y, t)$ and subscripts represent partial differentiation with respect to the given variable. This equation was used to describe the $(2+1)$-dimensional interaction of the Riemann wave propagated along the $\mathrm{y}$-axis with a long wave propagated along the $\mathrm{x}$-axis. The Painlevé analysis, Lax pairs, Bäcklund transformation, symmetry, similarity reductions and new exact solutions of the $(2+1)$-dimensional BLMP equation are given in [2-4]. In [5], based on the binary Bell polynomials, the bilinear form for the BLMP equation is obtained. New solutions of $(2+1)$-dimensional BLMP equation from Wronskian formalism and the Hirota method are obtained in [6,7].

The $(3+1)$-dimensional BLMP equation

$$
\begin{aligned}
& u_{y t}+u_{z t}+u_{x x x y}+u_{x x x z}-3 u_{x}\left(u_{x y}+u_{x z}\right) \\
& -3 u_{x x}\left(u_{y}+u_{z}\right)=0
\end{aligned}
$$

which was introduced in [8] has the bilinear form

$$
\left(D_{y} D_{t}+D_{z} D_{t}+D_{y} D_{x}^{3}+D_{z} D_{x}^{3}\right) f \cdot f=0
$$

just by substituting $u=-2(\ln f(x, y, z, t))_{x}$ into equation (2), where the bilinear differential operator $D$ is defined by Hirota [9] as

$$
\begin{aligned}
& D_{t}^{m} D_{x}^{n} a(t, x) \cdot b(t, x) \\
& =\left.\frac{\partial^{m}}{\partial s^{m}} \frac{\partial^{n}}{\partial y^{n}} a(t+s, x+y) b(t-s, x-y)\right|_{s=0, y=0}
\end{aligned}
$$

\section{Wronskian Formulation}

Solutions determined by $u=-2(\ln f)_{x}$ to the Equation (2) are called Wronskian solutions, where

$$
\begin{aligned}
f & =W\left(\phi_{1}, \phi_{2}, \cdots, \phi_{N}\right)=|\widehat{N-1}| \\
& =\left[\begin{array}{cccc}
\phi_{1}^{(0)} & \phi_{1}^{(1)} & \cdots & \phi_{1}^{(N-1)} \\
\phi_{2}^{(0)} & \phi_{2}^{(1)} & \cdots & \phi_{2}^{(N-1)} \\
\vdots & \vdots & \ddots & \vdots \\
\phi_{N}^{(0)} & \phi_{N}^{(1)} & \cdots & \phi_{N}^{(N-1)}
\end{array}\right], N>1,
\end{aligned}
$$

and

$$
\phi_{i}^{(0)}=\phi_{i}, \phi_{i}^{(j)}=\frac{\partial^{2}}{\partial x^{j}} \phi_{i}, j \geq 1,1 \leq i \leq N .
$$




\section{Lemma 1}

$|D, a, b||D, c, d|-|D, a, c||D, b, d|+|D, a, d||D, b, c|=0$, (6) where $D$ is $N \times(N-2)$ matrix, and $a, b, c, d$ are $n$-dimensional column vectors.

Lemma 2 Set $b_{j}(j=1, \cdots, n)$ to be an n-dimensional column vector, and $r_{j}(j=1, \cdots, n)$ to be a real constant but not to be zero. Then we have

$$
\sum_{i=1}^{N} r_{i}\left|b_{1}, b_{2}, \cdots, b_{N}\right|=\sum_{j=1}^{N}\left|b_{1}, b_{2}, \cdots, r b_{j}, \cdots, a_{N}\right|,
$$

where $r b_{j}=\left(r_{1} b_{1 j}, r_{2} b_{2 j}, \cdots, r_{N} b_{N j}\right)^{\mathrm{T}}$.

Lemma 3 The following equalities hold:

$$
\begin{aligned}
& f_{z}=f_{y}=f_{x}=|\widehat{N-2}, N|, \\
& f_{x z}=f_{x y}=f_{x x}=|\widehat{N-3}, N-1, N|+|\widehat{N-2}, N+1|, \\
& f_{x x z}=f_{x x y}=f_{x x x}=|\widehat{N-4}, N-2, N-1, N|+2|\widehat{N-3}, N-1, N+1|+|\widehat{N-2}, N+2| \\
& f_{x x x z}=f_{x x x y}=f_{x x x x}=|\widehat{N-5}, N-3, N-2,, N-1, N|+3|\widehat{N-4}, N-2, N-1, N+1| \\
& \quad+3|\widehat{N-3}, N-1, N+2|+2|\widehat{N-3}, N, N+1|+|\widehat{N-2}, N+3| \\
& f_{t}=-(4|\widehat{N-4}, N-2, N-1, N|-4|\widehat{N-3}, N-1, N+1|+4|\widehat{N-2}, N+2|), \\
& f_{y t}=f_{z t}=f_{x t}=-(4|\widehat{N-5}, N-3, N-2, N-1, N|-4|\widehat{N-3}, N, N+1|+4|\widehat{N-2}, N+3|) .
\end{aligned}
$$

Proof. Using the conditions (9), we get that

Hence, we have

$$
\begin{aligned}
& \left(D_{y} D_{t}+D_{z} D_{t}+D_{y} D_{x}^{3}+D_{z} D_{x}^{3}\right) f \cdot f \\
= & 6|\widehat{N-1}|(|\widehat{N-5}, N-3,, N-2,, N-1, N| \\
& -|\widehat{N-4}, N-2, N-1, N+1|-2|\widehat{N-3}, N, N+1| \\
& -|\widehat{N-3}, N-1, N+2|+|\widehat{N-2}, N+3|) \\
& +24|\widehat{N-3}, N-1, N+1||\widehat{N-2}, N| \\
& -6(|\widehat{N-3}, N-1, N|+|\widehat{N-2}, N+1|)^{2} .
\end{aligned}
$$

With the help of Lemma 2 and Lemma 3, we obtain

$$
\begin{aligned}
& 6|\widehat{N-1}|(|\widehat{N-5}, N-3, N-2, N-1, N| \\
& -|\widehat{N-4}, N-2, N-1, N+1|+2|\widehat{N-3}, N, N+1| \\
& -|\widehat{N-3}, N-1, N+2|+|\widehat{N-2}, N+3|) \\
& =6(-|\widehat{N-3}, N-1, N|+|\widehat{N-2}, N+1|)^{2} .
\end{aligned}
$$

Substituting Equation (11) into Equation (10) and using lemma 1, we get

$$
\begin{aligned}
& \left(D_{y} D_{t}+D_{z} D_{t}+D_{y} D_{x}^{3}+D_{z} D_{x}^{3}\right) f \cdot f \\
& =-24(|\widehat{N-3}, N-2, N+1||\widehat{N-3}, N-1, N| \\
& -|\widehat{N-3}, N-2, N-1||\widehat{N-3}, N+1, N| \\
& +|\widehat{N-3}, N-2, N||\widehat{N-3}, N+1, N-1|)=0 .
\end{aligned}
$$

Therefore, we have shown that $f=|\widehat{N-1}|$ solves Equation (4) under the linear differential conditions (9), The corresponding solution of Equation (2) is

$$
u=-2 \frac{f_{x}}{f}=-2 \frac{|\widehat{N-2}, N|}{|\widehat{N-1}|} \text {. }
$$

\section{Wronskian Solutions}

In what follows, according to [10-12], we would like to present a few special Wronskian solutions to the $(3+$ 
1)-dimensional Boiti-Leon-Manna-Pempinelli equation by solving the linear conditions (9).

It is well known that the corresponding Jordan form of a real marix

$$
A=\left[\begin{array}{cccc}
J\left(\lambda_{1}\right) & & & 0 \\
1 & J\left(\lambda_{2}\right) & & \\
& \ddots & \ddots & \\
0 & & 1 & J\left(\lambda_{m}\right)
\end{array}\right]_{N \times N},
$$

have the following two type of blocks:

1)

$$
J\left(\lambda_{i}\right)=\left[\begin{array}{cccc}
\lambda_{i} & & & 0 \\
1 & \lambda_{i} & & \\
& \ddots & \ddots & \\
0 & & 1 & \lambda_{i}
\end{array}\right]_{k_{i} \times k_{i}},
$$

2)

$$
\begin{gathered}
J\left(\lambda_{i}\right)=\left[\begin{array}{cccc}
A_{i} & & & 0 \\
I_{2} & A_{i} & & \\
& \ddots & \ddots & \\
0 & & I_{2} & A_{i}
\end{array}\right]_{l_{i} \times l_{i}}, \\
A_{i}=\left[\begin{array}{cc}
\alpha_{i} & -\beta_{i} \\
\beta_{i} & \alpha_{i}
\end{array}\right], I_{2}=\left[\begin{array}{ll}
1 & 0 \\
0 & 1
\end{array}\right],
\end{gathered}
$$

where $\lambda_{i}, \alpha_{i}$ and $\beta_{i}$ are all real constants. The first type of blocks have the real eigenvalue $\lambda_{i}$ with algebraic multiplicity $k_{i}\left(\sum_{i=1}^{n} k_{i}=N\right)$, and the second type of blocks have the complex eigenvalue $\lambda_{i}^{ \pm}=\alpha_{i} \pm \beta_{i} \sqrt{-1}$ with algebraic multiplicity $l_{i}$.

\subsection{Rational Solutions}

Suppose $A$ have the first type of Jordan blocks

$$
A=\left[\begin{array}{cccc}
\lambda_{1} & & & 0 \\
1 & \lambda_{1} & & \\
& \ddots & \ddots & \\
0 & & 1 & \lambda_{1}
\end{array}\right]_{N \times N},
$$

In this case, if the eigenvalue $\lambda_{1}=0$, corresponding to the following form:

$$
A=\left[\begin{array}{cccc}
0 & & & 0 \\
1 & 0 & & \\
& \ddots & \ddots & \\
0 & & 1 & 0
\end{array}\right]_{N \times N}
$$

from the condition (9), we get

$$
\phi_{i, x x}=0, \phi_{i, t}=-4 \phi_{i, x x x}, \phi_{i, y}=\phi_{i, x}, \phi_{i, z}=\phi_{i, x}, i \geq 1 \text {. }
$$

where $\phi_{i}(i \geq 1)$ are all polynomials in $x, y, z$ and $t$, and a general Wronskian solution to the $(3+1)$ dimen- sional Boiti-Leon-Manna-Pempinelli Equation (2)

$$
u=-2 \partial_{x} \ln W\left(\phi_{1}, \phi_{2}, \cdots, \phi_{k_{1}}\right),
$$

is called a rational Wronskian solution.

From Equation (18), we get

$$
\phi_{1, x x}=0, \phi_{1, t}=-4 \phi_{i, x x x}, \phi_{1, y}=\phi_{1, x}, \phi_{1, z}=\phi_{1, x} .
$$

Solving Equation (20) by using Maple, we get the following formulas:

$$
\phi_{1}=C_{1}(x+y+z)+C_{2} .
$$

Similarly, by solving

$$
\begin{aligned}
& \phi_{i+1, x x}=0, \phi_{i+1, t}=-4 \phi_{i+1, x x x}, \\
& \phi_{i+1, y}=\phi_{i+1, x}, \phi_{i+1, z}=\phi_{i+1, x}, i \geq 1,
\end{aligned}
$$

then two special rational solution of lower-order are obtained after setting some integral constants to be zero.

1) Zero-order: Taking $\phi_{1}=C_{1}(x+y+z)+C_{2}$, the corresponding Wronskian determinant and the associated rational Wronskian solution of zero-order read

$$
\begin{gathered}
f=W\left(\phi_{1}\right)=\phi_{1}=C_{1}(x+y+z)+C_{2}, \\
u=-2 \partial_{x} \ln W\left(\phi_{1}\right)=-\frac{2 C_{1}}{C_{1}(x+y+z)+C_{2}},
\end{gathered}
$$

where $C_{1}, C_{2}$ are arbitrary constants.

2) First-order: Taking $\phi_{1}=C_{1}(x+y+z)+C_{2}$, we can have

$$
\begin{aligned}
\phi_{2}= & \frac{C_{1}}{6}\left(x^{3}+(3 z+3 y) x^{2}+3(y+z)^{2} x-24 t+z^{3}+3 z y^{2}\right. \\
& \left.+3 z^{2} y+y^{3}\right)+\frac{1}{2} C_{2} x^{2}+\frac{1}{6}\left(6 C_{2} z+6 C_{3}+6 C_{2} y\right) x \\
& +\frac{1}{2} C_{2} y^{2}+\frac{1}{6}\left(6 C_{2} z+6 C_{3} y\right)+C_{4}+\frac{1}{2} C_{2} z^{2}+C_{3} z .
\end{aligned}
$$

Then, the corresponding Wronskian determinant and rational Wronskian solution of first-order are

$$
\begin{aligned}
f= & W\left(\phi_{1}, \phi_{2}\right)=P, \\
u=-2 \partial_{x} \ln W\left(\phi_{1}, \phi_{2}\right) & \frac{C_{1}^{2}\left(2 y z+2 x y+2 x z+x^{2}+y^{2}+z^{2}\right)}{P} \\
= & +\frac{2 C_{1} C_{2}(x+y+z)+C_{2}^{2}}{P},
\end{aligned}
$$

where

$$
\begin{aligned}
P= & C_{1}^{2}\left(x y^{2}+x z^{2}+x^{2} z+x^{2} y+z^{2} y+z y^{2}+2 x y z+\frac{1}{3} x^{3}\right. \\
& \left.+\frac{1}{3} y^{3}+\frac{1}{3} z^{3}+4 t\right)+C_{1} C_{2}(2 x y+2 y z+2 x y \\
& \left.+x^{2}+y^{2}+z^{2}\right)+C_{2}^{2}(x+y+z)+C_{2} C_{3}-C_{1} C_{4}
\end{aligned}
$$


and $C_{1}, C_{2}, C_{3}, C_{4}$ are arbitrary real constants. Similarly, we can obtain more higher order rational Wronskian solutions.

\subsection{Solitons, Negatons and Positons}

\subsubsection{Solitons}

If $A$ becomes to the following form

$$
A=\left[\begin{array}{cccc}
\lambda_{1} & & & 0 \\
& \lambda_{2} & & \\
& \ddots & \ddots & \\
0 & & & \lambda_{N}
\end{array}\right]_{N \times N},
$$

where the eigenvaluce $\lambda_{i} \neq 0$. Substituting the form of expression (26) into Equation (9), the following system of differential equations is obtained

$$
\begin{aligned}
& \left(\phi_{i}\left(\lambda_{i}\right)\right)=\lambda_{i}\left(\phi_{i}\left(\lambda_{i}\right)\right),\left(\phi_{i}\left(\lambda_{i}\right)\right)_{t}=-4\left(\phi_{i}\left(\lambda_{i}\right)\right)_{x x x}, \\
& \left(\phi_{i}\left(\lambda_{i}\right)\right)_{y}=\left(\phi_{i}\left(\lambda_{i}\right)\right)_{x},\left(\phi_{i}\left(\lambda_{i}\right)\right)_{z}=\left(\phi_{i}\left(\lambda_{i}\right)\right)_{x},
\end{aligned}
$$

By solving system (27), we get the $n$-soliton solution of Equation (2)

$$
u=-2 \partial_{x} \ln W\left(\phi_{1}, \phi_{2}, \cdots, \phi_{N}\right),
$$

with $\phi_{i}$ being defined by

$$
\begin{aligned}
& \phi_{i}=\cosh \left(\sqrt{\lambda_{i}} x+\sqrt{\lambda_{i}} y+\sqrt{\lambda_{i}} z-4 \lambda_{i}^{\frac{3}{2}} t\right), \quad i \text { odd } \\
& \phi_{i}=\sinh \left(\sqrt{\lambda_{i}} x+\sqrt{\lambda_{i}} y+\sqrt{\lambda_{i}} z-4 \lambda_{i}^{\frac{3}{2}} t\right), \quad i \text { even }
\end{aligned}
$$

where $0<\lambda_{1}<\lambda_{2}<\cdots<\lambda_{N}$ are arbitrary constants.

We present the 1 -soliton and 2-soliton solutions

$$
\begin{aligned}
& u_{1}=-2 \partial_{x} \ln \left(\cosh \left(\sqrt{\lambda_{1}} x+\sqrt{\lambda_{1}} y+\sqrt{\lambda_{1}} z-4 \lambda_{1}^{\frac{3}{2}} t\right)\right) \\
&=-2 \sqrt{\lambda_{1}} \tanh \left(\sqrt{\lambda_{1}} x+\sqrt{\lambda_{1}} y+\sqrt{\lambda_{1}} z-4 \lambda_{1}^{\frac{3}{2}} t\right) \\
& u_{2}=-2 \partial_{x} \ln W\left(\cosh \left(\sqrt{\lambda_{1}} x+\sqrt{\lambda_{1}} y+\sqrt{\lambda_{1}} z-4 \lambda_{1}^{\frac{3}{2}} t\right),\right. \\
&\left.\sinh \left(\sqrt{\lambda_{2}} x+\sqrt{\lambda_{2}} y+\sqrt{\lambda_{2}} z-4 \lambda_{2}^{\frac{3}{2}} t\right)\right) \\
&=\frac{2\left(\lambda_{1}-\lambda_{2}\right)}{P-Q}
\end{aligned}
$$

where

$$
\begin{aligned}
& P=\sqrt{\lambda_{2}} \operatorname{coth}\left(\sqrt{\lambda_{2}} x+\sqrt{\lambda_{2}} y+\sqrt{\lambda_{2}} z-4 \lambda_{2}^{\frac{3}{2}} t\right) \\
& Q=\sqrt{\lambda_{1}} \tanh \left(\sqrt{\lambda_{1}} x+\sqrt{\lambda_{1}} y+\sqrt{\lambda_{1}} z-4 \lambda_{1}^{\frac{3}{2}} t\right)
\end{aligned}
$$

Similarly, we can obtain 3-soliton, 4-soliton solution and $n$-soliton.

\subsubsection{Negatons and Positons}

If the eigenvalue $\lambda_{1} \neq 0, J\left(\lambda_{1}\right)$ becomes to the following form

$$
J\left(\lambda_{1}\right)=\left[\begin{array}{cccc}
\lambda_{1} & & & 0 \\
1 & \lambda_{1} & & \\
& \ddots & \ddots & \\
0 & & 1 & \lambda_{1}
\end{array}\right]_{k_{1} \times k_{1}},
$$

We start from the eigenfuction $\phi_{1}\left(\lambda_{1}\right)$, which is determined by

$$
\begin{aligned}
& \left(\phi_{1}\left(\lambda_{1}\right)\right)=\lambda_{1}\left(\phi_{1}\left(\lambda_{1}\right)\right),\left(\phi_{1}\left(\lambda_{1}\right)\right)_{t}=-4\left(\phi_{1}\left(\lambda_{1}\right)\right)_{x x x}, \\
& \left(\phi_{1}\left(\lambda_{1}\right)\right)_{y}=\left(\phi_{1}\left(\lambda_{1}\right)\right)_{x},\left(\phi_{1}\left(\lambda_{1}\right)\right)_{z}=\left(\phi_{1}\left(\lambda_{1}\right)\right)_{x},
\end{aligned}
$$

General solution to this system in two cases of $\lambda_{1}>0$ and $\lambda_{1}<0$ are

$$
\begin{aligned}
& \phi_{1}\left(\lambda_{1}\right)=C_{1} \cosh \left(\sqrt{\lambda_{1}} x+\sqrt{\lambda_{1}} y+\sqrt{\lambda_{1}} z-4 \lambda_{1}^{\frac{3}{2}} t\right) \\
& +C_{2} \sinh \left(\sqrt{\lambda_{1}} x+\sqrt{\lambda_{1}} y+\sqrt{\lambda_{1}} z-4 \lambda_{1}^{\frac{3}{2}} t\right), \lambda_{1}>0, \\
& \phi_{1}\left(\lambda_{1}\right)=C_{3} \cos \left(\sqrt{-\lambda_{1}} x+\sqrt{-\lambda_{1}} y+\sqrt{-\lambda_{1}} z+4\left(-\lambda_{1}\right)^{\frac{3}{2}} t\right) \\
& +C_{4} \sin \left(\sqrt{-\lambda_{1}} x+\sqrt{-\lambda_{1}} y+\sqrt{-\lambda_{1}} z+4\left(-\lambda_{1}\right)^{\frac{3}{2}} t\right), \lambda_{1}<0,
\end{aligned}
$$

respectively, where $C_{1}, C_{2}, C_{3}$ and $C_{4}$ are arbitrary real constants. When $\lambda_{1}>0$, we get negaton solution and when $\lambda_{1}<0$, we get positon solutions.

To construct Wronskian solutions corresponding to Jordan blocks of higher-order, we use the basic idea developed for the $\mathrm{KdV}$ equation $[10,11]$.

Differentiating (9) with respect to $\lambda_{1}$, we can find that the vector function

$$
\begin{aligned}
\Phi_{1} & =\Phi_{1}\left(\lambda_{1}\right) \\
& =\left(\phi_{1}\left(\lambda_{1}\right), \frac{1}{1 !} \partial_{\lambda_{1}} \phi_{1}\left(\lambda_{1}\right), \cdots, \frac{1}{\left(k_{1}-1\right) !} \partial_{\lambda_{1}}^{k_{1}-1} \phi_{1}\left(\lambda_{1}\right)\right)^{\mathrm{T}},
\end{aligned}
$$

satisfies

$$
\Phi_{1, x x x}=\left[\begin{array}{cccc}
\lambda_{1} & & & 0 \\
1 & \lambda_{1} & & \\
& \ddots & \ddots & \\
0 & & 1 & \lambda_{1}
\end{array}\right]_{k_{1} \times k_{1}} \Phi_{1}
$$




$$
\Phi_{1, t}=-4 \Phi_{1, x x x}, \Phi_{1, y}=\Phi_{1, x}, \Phi_{1, z}=\Phi_{1, x}
$$

where $\partial_{\lambda_{1}}$ denotes the derivative with respect to $\lambda_{1}$ and $k_{1}$ is an arbitrary nonnegative integer. Therefore, through this set of eigenfunctions and Equation (12), a Wronskian solution of order $k_{1}-1$ to Equation (2) is presented as: u

$=-2 \partial_{x} \ln W\left(\phi_{1}\left(\lambda_{1}\right), \frac{1}{1 !} \partial_{\lambda_{1}} \phi_{1}\left(\lambda_{1}\right), \cdots, \frac{1}{\left(k_{1}-1\right) !} \partial_{\lambda_{1}}^{k_{1}-1} \phi_{1}\left(\lambda_{1}\right)\right)$, which corresponds to the first type of Jordan blocks with a nonzero real eigenvalue.

In what follows, several exact solutions of lower-order are presented to the $(3+1)$-dimensional Boiti-LeonManna-Pempinelli equation as where

$$
\begin{gathered}
\eta_{1}=\sqrt{\lambda_{1}} x+\sqrt{\lambda_{1}} y+\sqrt{\lambda_{1}} z-4 \lambda_{1}^{\frac{3}{2}} t . \\
\theta_{1}=\sqrt{-\lambda_{1}} x+\sqrt{-\lambda_{1}} y+\sqrt{-\lambda_{1}} z+4\left(-\lambda_{1}\right)^{\frac{3}{2}} t .
\end{gathered}
$$

$$
\begin{aligned}
& u_{1 \text {-negaton }}=-2 \partial_{x} \ln \left(\cosh \left(\sqrt{\lambda_{1}} x+\sqrt{\lambda_{1}} y+\sqrt{\lambda_{1}} z-4 \lambda_{1}^{\frac{3}{2}} t\right)\right) \\
&=-2 \sqrt{\lambda_{1}} \tanh \left(\sqrt{\lambda_{1}} x+\sqrt{\lambda_{1}} y+\sqrt{\lambda_{1}} z-4 \lambda_{1}^{\frac{3}{2}} t\right), \\
& u_{1 \text {-positon }}=-2 \partial_{x} \ln \left(\cos \left(\sqrt{-\lambda_{1}} x+\sqrt{-\lambda_{1}} y+\sqrt{-\lambda_{1}} z+4\left(-\lambda_{1}\right)^{\frac{3}{2}} t\right)\right) \\
&= 2 \sqrt{-\lambda_{1}} \tan \left(\sqrt{-\lambda_{1}} x+\sqrt{-\lambda_{1}} y+\sqrt{-\lambda_{1}} z+4\left(-\lambda_{1}\right)^{\frac{3}{2}} t\right), \\
& u_{2 \text {-negaton }}=-2 \partial_{x} \ln W\left(\cosh \left(\eta_{1}\right), \partial_{\lambda_{1}} \cosh \left(\eta_{1}\right)\right) \\
&= \frac{4 \sqrt{\lambda_{1}} \cosh \left(\eta_{1}\right)}{}-\cosh \left(\eta_{1}\right) \sinh \left(\eta_{1}\right)-\sqrt{\lambda_{1}} x-\sqrt{\lambda_{1}} y-\sqrt{\lambda_{1}} z+12 \lambda_{1}^{\frac{3}{2}} t \\
&=-2 \partial_{x} \ln W\left(\cos \left(\theta_{1}\right), \partial_{\lambda_{1}} \cos \left(\theta_{1}\right)\right) \\
& 4 \lambda_{1} \cosh \left(\theta_{1}\right) \\
&= \frac{\sqrt{-\lambda_{1}} \cos \left(\theta_{1}\right) \sin \left(\theta_{1}\right)-\lambda_{1} x-\lambda_{1} y-\lambda_{1} z+12 \lambda_{1}^{\frac{3}{2}} t}{u_{2-\text { positon }}},
\end{aligned}
$$

\subsection{Interaction Solutions}

We are now presenting examples of Wronskian interaction solutions among different kinds of Wronskian solutions to the $(3+1)$-dimensional Boiti-Leon-MannaPempinelli equation.

Let us assume that there are two sets of eigenfunctions

$$
\phi_{1}(\lambda), \phi_{2}(\lambda), \cdots, \phi_{k}(\lambda) ; \psi_{1}(\mu) \psi_{2}(\mu), \cdots, \psi_{l}(\mu),
$$

associated with two different eigenvalues $\lambda$ and $\mu$, respectively. A Wronskian solution

$$
\begin{gathered}
u=-2 \partial_{x}^{2} \ln W\left(\phi_{1}(\lambda), \phi_{2}(\lambda), \cdots, \phi_{k}(\lambda) ; \psi_{1}(\mu)\right. \\
\left.\psi_{2}(\mu), \cdots, \psi_{l}(\mu)\right)
\end{gathered}
$$

is said to be a Wronskian interaction solution between two solutions determined by the two sets of eigenfunc- tions in (37). In fact, we can have more general Wronskian interaction solutions among three or more kinds of solutions such as rational solutions, positons, solitons, negatons, breathers and complexitons.

In what follows, we would like to show a few special Wronskian interaction solutions depending on rational solution, positons and solitons. Firstly, we choose three different sets of special eigenfunctions:

$$
\begin{aligned}
& \phi_{\text {rational }}=x+y+z, \\
& \phi_{\text {soliton }}=\cosh \left(\sqrt{\lambda_{1}} x+\sqrt{\lambda_{1}} y+\sqrt{\lambda_{1}} z-4 \lambda_{1}^{\frac{3}{2}} t\right), \\
& \phi_{\text {positon }}=\cos \left(\sqrt{-\lambda_{2}} x+\sqrt{-\lambda_{2}} y+\sqrt{-\lambda_{2}} z+4\left(-\lambda_{2}\right)^{\frac{3}{2}} t\right),
\end{aligned}
$$

where $\lambda_{1}>0, \lambda_{2}<0$ are constants.

Three Wronskian interaction determinants between any two of a rational solution, a single soliton and a single positon are obtained as 


$$
\begin{aligned}
& W\left(\phi_{\text {rational }}, \phi_{\text {soliton }}\right) \\
& =\sqrt{\lambda_{1}}(x+y+z) \sinh \left(\eta_{1}\right)-\cosh \left(\eta_{1}\right), \\
& W\left(\phi_{\text {rational }}, \phi_{\text {positon }}\right) \\
& =-\sqrt{-\lambda_{2}}(x+y+z) \sin \left(\eta_{2}\right)-\cos \left(\eta_{2}\right) \\
& W\left(\phi_{\text {soliton }}, \phi_{\text {positon }}\right) \\
& =-\sqrt{-\lambda_{2}} \cosh \left(\eta_{1}\right) \sinh \left(\eta_{2}\right)-\sqrt{\lambda_{1}} \cos \left(\eta_{2}\right) \sinh \left(\eta_{1}\right),
\end{aligned}
$$

where

$$
\begin{gathered}
\eta_{1}=\sqrt{\lambda_{1}} x+\sqrt{\lambda_{1}} y+\sqrt{\lambda_{1}} z-4 \lambda_{1}^{\frac{3}{2}} t \\
\eta_{2}=\sqrt{-\lambda_{1}} x+\sqrt{-\lambda_{1}} y+\sqrt{-\lambda_{1}} z+4\left(-\lambda_{1}\right)^{\frac{3}{2}} t .
\end{gathered}
$$

Further, the corresponding Wronskian interaction solutions are

$$
\begin{gathered}
u_{r s}=-2 \partial_{x} \ln W\left(\phi_{\text {rational }}, \phi_{\text {soliton }}\right) \\
=\frac{2 \lambda_{1}(x+y+z) \cosh \left(\eta_{1}\right)}{\sqrt{\lambda_{1}}(x+y+z) \sinh \left(\eta_{1}\right)-\cosh \left(\eta_{1}\right)}, \\
u_{r p}=-2 \partial_{x} \ln W\left(\phi_{\text {rational }}, \phi_{\text {positon }}\right) \\
=\frac{2 \lambda_{2}(x+y+z) \cos \left(\eta_{2}\right)}{-\sqrt{-\lambda_{2}}(x+y+z) \sin \left(\eta_{2}\right)-\cos \left(\eta_{2}\right)} \\
u_{s p}=-2 \partial_{x} \ln W\left(\phi_{\text {soliton }}, \phi_{\text {positon }}\right) \\
=\frac{2\left(\lambda_{2}-\lambda_{1}\right) \cosh \left(\eta_{1}\right) \cos \left(\eta_{2}\right)}{-\sqrt{-\lambda_{2}} \cosh \left(\eta_{1}\right) \sinh \left(\eta_{2}\right)-\sqrt{\lambda_{1}} \cos \left(\eta_{2}\right) \sinh \left(\eta_{1}\right)},
\end{gathered}
$$

where

$$
\begin{gathered}
\eta_{1}=\sqrt{\lambda_{1}} x+\sqrt{\lambda_{1}} y+\sqrt{\lambda_{1}} z-4 \lambda_{1}^{\frac{3}{2}} t, \\
\eta_{2}=\sqrt{-\lambda_{2}} x+\sqrt{-\lambda_{2}} y+\sqrt{-\lambda_{2}} z+4\left(-\lambda_{2}\right)^{\frac{3}{2}} t .
\end{gathered}
$$

The following is one Wronskian interaction determinant and solution involving the three eigenfunctions. The Wronskian determinant is

$$
\begin{aligned}
& W\left(\phi_{\text {rational }}, \phi_{\text {soliton }}, \phi_{\text {positon }}\right) \\
& =(x+y+z)\left(\lambda_{2} \sqrt{\lambda_{1}} \sinh \left(\eta_{1}\right) \cos \left(\eta_{2}\right)\right. \\
& \left.\quad+\lambda_{1} \sqrt{-\lambda_{2}} \sin \left(\eta_{2}\right) \cosh \left(\eta_{1}\right)\right),
\end{aligned}
$$

so that its corresponding Wronskian solution reads as

$$
u_{r s p}=-2 \partial_{x} \ln W\left(\phi_{\text {rational }}, \phi_{\text {soliton }}, \phi_{\text {positon }}\right)=\frac{-2 q_{3}}{p_{3}},
$$

where

$$
\begin{aligned}
& p_{3}=(x+y+z)\left(\lambda_{2} \sqrt{\lambda_{1}} \sinh \left(\eta_{1}\right) \cos \left(\eta_{2}\right)\right. \\
& \left.+\lambda_{1} \sqrt{-\lambda_{2}} \sin \left(\eta_{2}\right) \cosh \left(\eta_{1}\right)\right), \\
& q_{3}=(x+y+z) \sqrt{-\lambda_{1} \lambda_{2}}\left(\lambda_{1}-\lambda_{2}\right) \sinh \left(\eta_{1}\right) \sin \left(\eta_{2}\right) \\
& +\lambda_{1} \sqrt{\lambda_{1}} \sinh \left(\eta_{1}\right) \cos \left(\eta_{2}\right)+\lambda_{2} \sqrt{-\lambda_{2}} \cosh \left(\eta_{1}\right) \sin \left(\eta_{2}\right)
\end{aligned}
$$

with

$$
\begin{gathered}
\eta_{1}=\sqrt{\lambda_{1}} x+\sqrt{\lambda_{1}} y+\sqrt{\lambda_{1}} z-4 \lambda_{1}^{\frac{3}{2}} t, \\
\eta_{2}=\sqrt{-\lambda_{2}} x+\sqrt{-\lambda_{2}} y+\sqrt{-\lambda_{2}} z+4\left(-\lambda_{2}\right)^{\frac{3}{2}} t .
\end{gathered}
$$

\section{Conclusion}

In this paper, by using the Wronskian technique, we have derived the Wronskian determinant solution for the $(3+$ 1)-dimensional Boiti-Leon-Manna-Pempinelli equation which describes the fluid propagating and can be considered as a model for an incompressible fluid. Moreover, we obtained some rational solutions, soliton solutions, positons and negatons of this equation by solving the resultant systems of linear partial differential equations which guarantee that the Wronskian determinant solves the equation in the bilinear form. The presented solutions show the remarkable richness of the solution space of the $(3+1)$-dimensional Boiti-Leon-Manna-Pempinelli equation.

\section{Acknowledgements}

The work is supported by National Natural Science Foundation of China (project No. 11371086), the Fund of Science and Technology Commission of Shanghai Municipality (project No. ZX201307000014) and the Fundamental Research Funds for the Central Universities.

\section{REFERENCES}

[1] N. C. Freeman and J. J. C. Nimmo, "Soliton Solutions of the Korteweg-de Vries and Kadomtsev-Petviashvili Equations: The Wronskian Technique,” Physics Letters A, Vol. 95, No. 1, 1983, pp. 1-3.

http://dx.doi.org/10.1016/0375-9601(83)90764-8

[2] M. Boiti, J. J.-P. Leon and F. Pempinelli, "On the Spectral Transform of a Korteweg-de Vries Equation in Two Spatial Dimensions," Inverse Problems, Vol. 2, No. 3, 1986, pp. 271-279.

http://dx.doi.org/10.1088/0266-5611/2/3/005

[3] C.-J. Bai and H. Zhao, "New Solitary Wave and Jacobi Periodic Wave Excitations in (2+1)-Dimensional BoitiLeon-Manna-Pempinelli System,” International Journal of Modern Physics B, Vol. 22, No. 15, 2008, pp. $2407-$ 2420. http://dx.doi.org/10.1142/S021797920803954X

[4] Y. Li and D. Li, "New Exact Solutions for the (2+1)Dimensional Boiti-Leon-Manna-Pempinelli Equation,” Ap- 
plied Mathematical Sciences, Vol. 6, No. 12, 2012, pp. 579-587.

[5] L. Luo, "New Exact Solutions and Bäcklund Transformation for Boiti-Leon-Manna-Pempinelli Equation,” Physics Letters A, Vol. 375, No. 7, 2011, pp. 1059-1063. http://dx.doi.org/10.1016/j.physleta.2011.01.009

[6] L. Delisle and M. Mosaddeghi, "Classical and SUSY Solutions of the Boiti-Leon-Manna-Pempinelli Equation," Journal of Physics A: Mathematical and Theoretical, Vol. 46, No. 11, 2013, Article ID: 115203. http://dx.doi.org/10.1088/1751-8113/46/11/115203

[7] M. Najafi and S. Arbabi, "Wronskian Determinant Solutions of the (2+1)-Dimensional Boiti-Leon-Manna-Pempinelli Equation," International Journal of Advanced Mathematical Sciences, Vol. 1, No. 1, 2013, pp. 8-11.

[8] M. Darvishi, M. Najafi, L. Kavitha and M. Venkatesh, "Stair and Step Soliton Solutions of the Integrable $(2+1)$ and (3+1)-Dimensional Boiti-Leon-Manna-Pempinelli Equations," Communications in Theoretical Physics, Vol.
58, No. 6, 2012, pp. 785-794. http://dx.doi.org/10.1088/0253-6102/58/6/01

[9] R. Hirota, “The Direct Method in Soliton Theory,” Cambridge University Press, Cambridge, 2004.

[10] W. X. Ma, "Wronskians, Generalized Wronskians and Solutions to the Korteweg-de Vries Equation," Chaos, Solitons and Fractals, Vol. 19, No. 1, 2004, pp. 163-170. http://dx.doi.org/10.1016/S0960-0779(03)00087-0

[11] W.-X. Ma and Y. You, "Solving the Korteweg-de Vries Equation by Its Bilinear Form: Wronskian Solutions," Transactions of the American Mathematical Society, Vol. 357, No. 5, 2005, pp. 1753-1778. http://dx.doi.org/10.1090/S0002-9947-04-03726-2

[12] C.-X. Li, W.-X. Ma, X.-J. Liu and Y.-B. Zeng, "Wronskian Solutions of the Boussinesq Equation-Solitons, Negatons, Positons and Complexitons,” Inverse Problems, Vol. 23, No. 1, 2007, pp. 279-296. http://dx.doi.org/10.1088/0266-5611/23/1/015 